\title{
A Multi-Objective Antenna Placement Genetic Algorithm for Matched Array Synthesis on Complex Platforms
}

\author{
Mythili.P, Pelumi E. Osoba,Eric Michielssen \\ Radiation Laboratory, EECS \\ University of Michigan \\ MI, USA \\ mythili@umich.edu,osoba@umich.edu, emichiel@umich.edu
}

\begin{abstract}
A Multi-Objective Antenna Placement Genetic Algorithm (MO-APGA) has been proposed for the synthesis of matched antenna arrays on complex platforms. The total number of antennas required, their position on the platform, location of loads, loading circuit parameters, decoupling and matching network topology, matching network parameters and feed network parameters are optimized simultaneously. The optimization goal was to provide a given minimum gain, specific gain discrimination between the main and back lobes and broadband performance. This algorithm is developed based on the non-dominated sorting genetic algorithm (NSGA-II) and Minimum Spanning Tree (MST) technique for producing diverse solutions when the number of objectives is increased beyond two. The proposed method is validated through the design of a wideband airborne $S A R$.
\end{abstract}

Keywords-Antenna placement; matched arrays; complex platforms; Genetic Algorithm; DMN; multi-objective.

\section{INTRODUCTION}

Positioning of multiple antennas on electrically large platforms is a complex problem. The performance of these antennas depends on the number of antenna elements, their physical location on the appropriate platform and the required matching network. Even though few algorithms and techniques [1-3] are available to simulate electrically large complex platforms, multi-antenna placement optimization still remains a difficult task. Optimizers, both stochastic and deterministic have limited ability to perform an exhaustive search for multiantenna configurations on such platforms without running out of computational resources. A two objective Antenna Placement Genetic Algorithm (APGA) [4] that utilizes a parallel non-dominated sorting genetic algorithm (NSGA-II) [5] as the optimizer was used to overcome this issue. This algorithm failed to produce diverse solutions as the number of objectives increased beyond two in addition to ignoring the complete antenna system design.

In this paper a Multi-Objective Antenna Placement Algorithm (MO-APGA) using the NSGA-II based on minimum spanning tree (NSGA-II+MST) [6] as optimizer has been proposed for the matched array synthesis on complex platforms. A decoupling and matching network (DMN) [7] and cable connector have been included to complete the system design. Specifically a single input multiple output (SIMO) DMN has been used for matching and decoupling purposes. Furthermore reduction in computational cost has been achieved by modifying certain stages in the optimization process without degrading the accuracy.

\section{MO-APGA DESCRIPTION}

MO-APGA is a modified version of the APGA [4]. While APGA uses only NSGA-II, MO-APGA uses the NSGAII+MST as optimizer. APGA failed to produce diverse solutions as the number of objectives increased beyond two [6]. This was due to the technique used by APGA to compute the crowding distance between the individuals in a population. Crowding distance is a measure which plays a significant role in obtaining diverse solutions in multi objective optimization problems. It provides an estimate of the density of the solutions surrounding a particular solution in the population. The NSGAII+MST algorithm overcomes the diversity issues by constructing a shortest spanning tree [8] over the entire population to estimate the density of the individuals. In addition to the change in optimizer the matching and decoupling network and the cable connecter has been included in the optimization process to improve the performance of the system.

Fig. 1 shows the block diagram of the complete antenna system to be optimized on the appropriate platform. $Z_{M}, Z_{C}$, and $\mathrm{Z}_{\mathrm{A}}$ are the impedances that represent the $\mathrm{DMN}$, cable connector and the Antenna array. Assuming $\mathrm{N}$ antennas are placed randomly on the platform, $\mathrm{Z}_{\mathrm{A}}$ is an $\mathrm{N} \times \mathrm{N}$ impedance

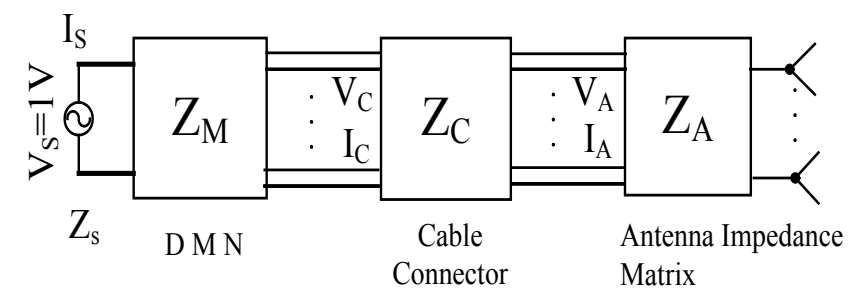

Figure 1. Antenna system block diagram 
matrix and $Z_{M}$ is an $(\mathrm{N}+1) \times(\mathrm{N}+1)$ matrix which includes the single input port of the DMN and the $\mathrm{N}$ output ports of the DMN. Further these impedance matrices are combined to form a single impedance matrix which represents the whole antenna system in the optimization process. The topology of a three port lossless SIMO DMN [7] which is used for matching the transmitting antennas with the source is shown in Fig.2. Since it is a lossless network, it uses only discrete reactive components.

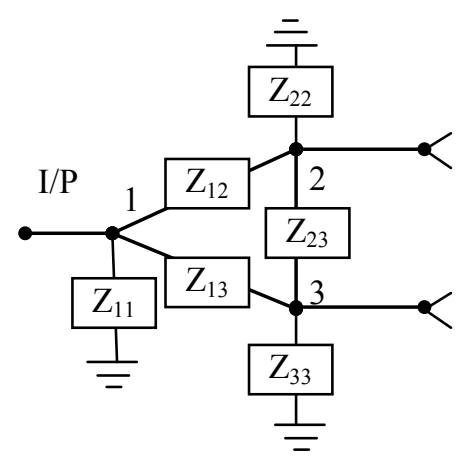

Figure 2. Topology of a three port SIMO DMN

The benefit of such a design is that only a single source is needed. The DMN is responsible for creating the phase delay required for beam forming as well as it reduces the coupling effects between the antennas randomly and non-uniformly positioned on the platform. The cable impedance matrix is used to represent the different transmission line lengths from the output of the DMN to the input of each one of the selected $\mathrm{N}$ antenna elements. Before starting the optimizer, the $\mathrm{N}$ port antenna impedance matrix and the full field data assuming the presence of all the antennas and their loads on the platform is obtained using a computational electromagnetic solver (CEM). The antenna selection / placement are performed by the MOAPGA. The size of the combined impedance matrix will depend on the number of antennas selected by the algorithm.

Each solution/individual in the population represents the number of antennas required, their position on the platform, location of loads, loading circuit components and their values, DMN topology and their components, values and feed network parameters. The MO-APGA optimizes them simultaneously to achieve a minimum gain requirement with specific gain discrimination between the main and back lobes and broadband performance.

\section{PROBLEM DESCRIPTION}

A fully matched loaded monopole antenna [3] array with suitable loads are to be optimally placed on an aircraft to provide a minimum gain requirement, $10 \mathrm{~dB}$ gain discrimination between the main and back lobe and broadband performance. The geometry of the aircraft used as platform, the possible antenna element locations on the aircraft and the desired main beam are shown in Fig.1 of [4]. The antenna design problem is completely defined by three objectives. MOAPGA has been used to find the optimal subset of platform sites for monopole placement, and to systematically place tank circuits at each monopole site to arrive at a fully matched array system satisfying the three objectives. A typical application of such a system is the airborne SAR.

\section{OPTIMIZATION CRITERIA}

A controlled multi-objective search with the help of the pareto-optimal candidates provides the designer with multiple design considerations. The binary chromosome encodes monopole selection, load locations, excitation voltage, elements of the DMN, cable length, and load component values for the selected monopole antennas. A triple objective function given by (1) - (3) is used to capture the entire design of the antenna system.

$$
\begin{aligned}
& \operatorname{maximize} f_{1}: \frac{1}{N} \sum \Upsilon_{s} \\
& \operatorname{minimize} f_{2}: \frac{1}{N} \sum\left(\operatorname{Gain}_{S L+B L}(\omega, \phi, \theta)\right) \\
& \operatorname{maximize} f_{3}: \frac{1}{N} \sum \operatorname{Gain}_{M L}(\omega, \phi, \theta)
\end{aligned}
$$

satisfying the following conditions.

$$
\begin{aligned}
& \Upsilon_{s}=1-\left|\Gamma_{s}\right|^{2} \\
& f_{1}: \forall \Upsilon_{s} \geq-1 d B, \Upsilon_{s}=-1 d B \\
& f_{2}: \forall \operatorname{Gain}_{S L+B L}(\omega, \phi, \theta) \leq-5 d B, \\
& \operatorname{Gain}_{S L+B L}(\omega, \phi, \theta)=-5 d B
\end{aligned}
$$

$$
\begin{aligned}
f_{3}: \forall \operatorname{Gain}_{M L}(\omega, \phi, \theta) & \geq 5 d B, \\
\operatorname{Gain}_{M L}(\omega, \phi, \theta) & =5 d B
\end{aligned}
$$

where SL,BL and ML refers to side lobe, main lobe and back lobe.

\section{NUMERICAL RESULTS}

The constraints specified by (1) - (3) were applied to the design of an aircraft mounted SAR for an operating frequency range of $140-160 \mathrm{MHz}$. The task was to select 4 monopoles from a set of 15 . The antenna system had to exhibit a directive main beam around $\Theta=135^{\circ}$ on the $\mathrm{YZ}$ plane and a $10 \mathrm{~dB}$ gain discrimination between the main lobe and the region surrounding the $\Theta=-135^{\circ}$ direction on the YZ plane. The VSWR, Gain discrimination, radiation pattern obtained using MO-AGPA is shown in Figs.3, 4 and 5. The three objective Pareto plot is shown in Fig.6 and the corresponding convergence graph is shown in Fig.7. The crossover and mutation rates were chosen as 0.85 and 0.001 . 


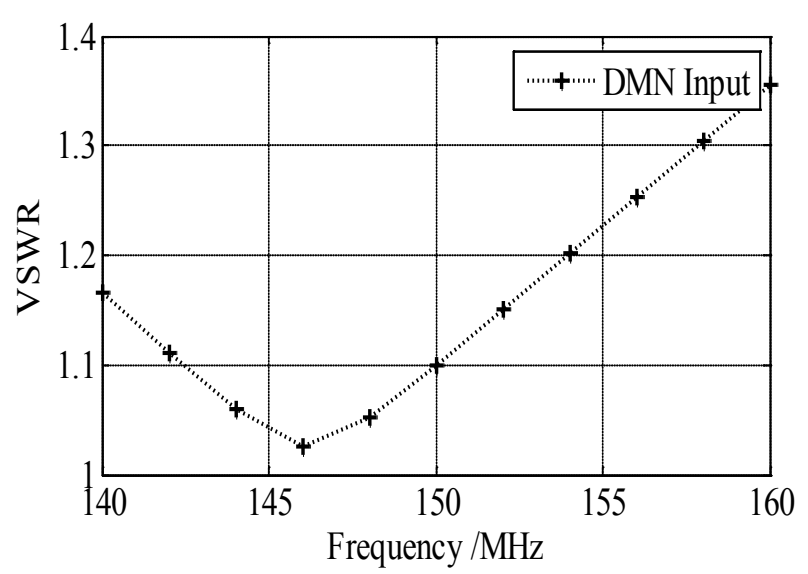

Figure 3. VSWR at source point

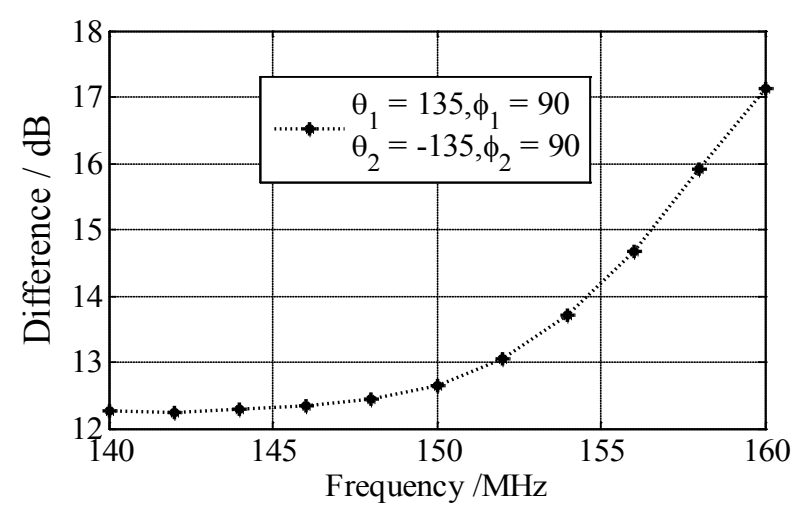

Figure 4. Gain discrimination

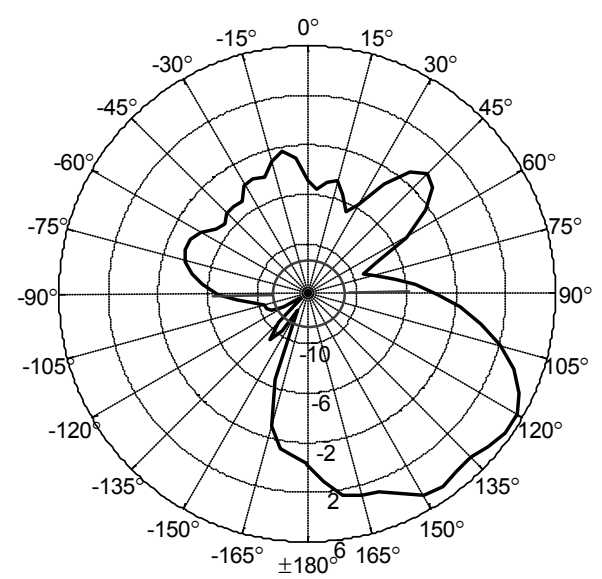

Figure 5. Gain/dBi in the Y-Z Plane (cut at the centre of the aircraft)

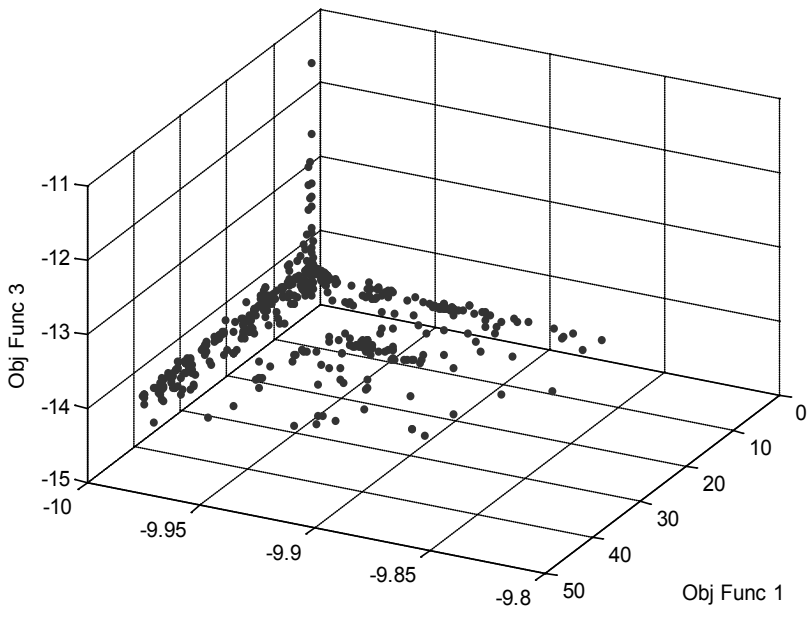

Obj Func 2

Figure 6. Three objective paretoplot

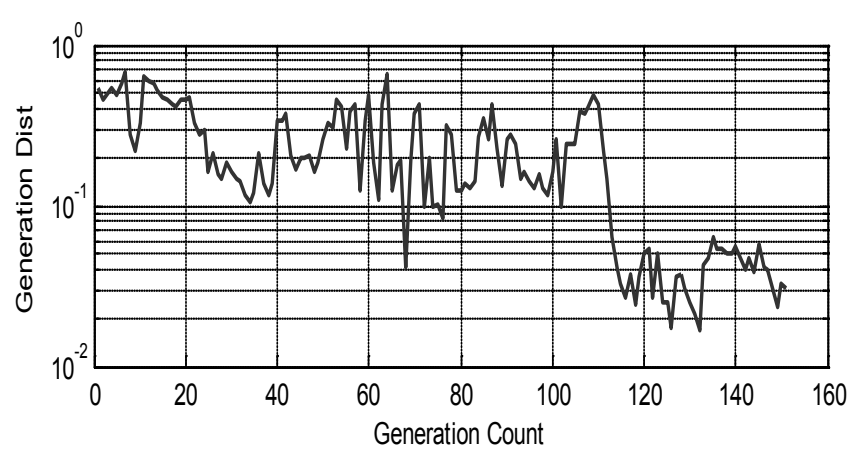

Figure 7. Paretofront convergence plot

From the results shown in Fig.3 to 7 and that of APGA [4], it can be seen that the MO-APGA performs better compared to the APGA in all aspects. A gain of around $6 \mathrm{dBi}$ could be achieved in the $\mathrm{Y}-\mathrm{Z}$ plane with a minimum $12 \mathrm{~dB}$ gain difference between the front and back lobes in the frequency range of $140-160 \mathrm{MHz}$. A bandwidth of $20 \mathrm{MHz}$ has been achieved.

\section{CONCLUSION}

A MO-APGA has been proposed for the positioning of multiple antennas on an aircraft for a given set of requirements. Unlike the earlier version it uses the MST for computing the crowding distance between the individuals in the population. It also includes the complete antenna system parameters in the design optimization process compared to APGA. The algorithm has been tested for 3 objectives and the results are promising. 


\section{ACKNOWLEDGMENT}

Mythili Palayyan thanks the Department of Science and Technology (DST), India for providing financial support under the BOYSCAST fellowship scheme. She thanks Prof. Eric Michielssen, University of Michigan for acting as her host and she acknowledges the facilities and other help provided by the University of Michigan Radiation Laboratory.

\section{REFERENCES}

[1] R.L.Haupt,"Thinned arrays using genetic Algorithms," IEEE Trans. Ant ennas Propag., vol.42, No.2, pp.993-999, Jul.1994.

[2] E.M.Koper, W.D.Wood, and S.W.Schneider, "Aircraft antenna coupling minimization using genetic algorithms and approximations,"IEEE Trans. Aerosp. Electron. Syst., vol.40,no.2,pp.742-751, Apr. 2004
[3] Daniel S.Weile and Eric Michielssen, "Genetic Algorithm Optimization Applied to Electromagnetics: A Review", IEEE Trans. Antennas and propagat., vol.45,no.3, pp343-353, Mar. 1997.

[4] P.E. Osoba, E. Michielssen. "Antenna Placement on Complex Platforms by Multi-Objective Genetic Algorithms," presented at Antennas and Propagation Society International Symposium, North Charleston, NC, June 2009.

[5] K. Deb, A. Pratap, S. Agarwal, and T. Meyarivan, "A fast and elitist multiobjective genetic algorithm: NSGA-II," IEEE Trans. Evol. Comput., vol.6, no.2, pp. 182-197, Apr. 2002.

[6] Miquing Li, Jinhua, and Jun Wu, “ Improving NSGA II Algorithm based on minimum spanning tree", Simulated Evolution and Learning, Berlin / Heidelberg: Springer Verlag, 2008, pp.170-179.

[7] Weber, J.; Volmer, C.; Blau, K.; Stephan, R.; Hein, M.A., "Miniaturized antenna arrays using decoupling networks with realistic elements," IEEE Trans. Microwave Theory and Tech., June 2006.

[8] Bang Ye Wu, Kun-Mao Chao, Spanning Trees and Optimization Problems, Chapman and Hall/CRC; 1 edition, 2004. 